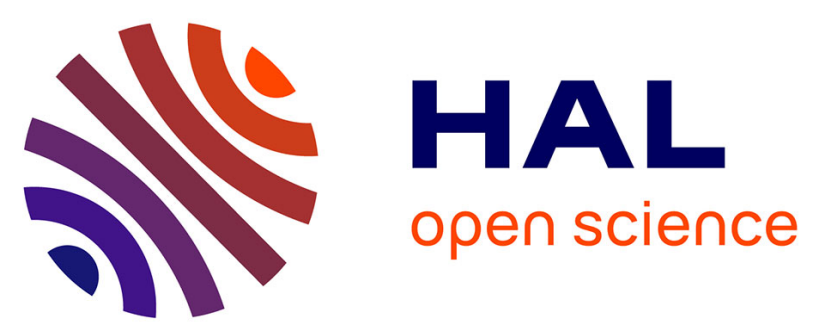

\title{
MODELLING OF THE BEHAVIOUR OF HOPPING CONDUCTIVITY IN A SOLID EXHIBITING A SEMICONDUCTOR-INSULATOR TRANSITION, AS A FUNCTION OF FREQUENCY
}

J. Giuntini, J. Jacquemin, J. Zanchetta, G. Bordure

\section{To cite this version:}

J. Giuntini, J. Jacquemin, J. Zanchetta, G. Bordure. MODELLING OF THE BEHAVIOUR OF HOPPING CONDUCTIVITY IN A SOLID EXHIBITING A SEMICONDUCTOR-INSULATOR TRANSITION, AS A FUNCTION OF FREQUENCY. Journal de Physique Colloques, 1981, 42 (C4), pp.C495-C4-98. 10.1051/jphyscol:1981416 . jpa-00220783

\section{HAL Id: jpa-00220783 https://hal.science/jpa-00220783}

Submitted on 1 Jan 1981

HAL is a multi-disciplinary open access archive for the deposit and dissemination of scientific research documents, whether they are published or not. The documents may come from teaching and research institutions in France or abroad, or from public or private research centers.
L'archive ouverte pluridisciplinaire HAL, est destinée au dépôt et à la diffusion de documents scientifiques de niveau recherche, publiés ou non, émanant des établissements d'enseignement et de recherche français ou étrangers, des laboratoires publics ou privés. 


\title{
MODELLING OF THE BEHAVIOUR OF HOPPING CONDUCTIVITY IN A SOLID EXHIBITING A SEMICONDUCTOR-INSULATOR TRANSITION, AS A FUNCTION OF
} FREQUENCY

\author{
J.C. Giuntini ${ }^{*}$, J.L. Jacquemin ${ }^{* *}$, J.V. Zanchetta* and G. Bordure J $^{*}$ \\ Université des Sciences et Techniques du Languedoc, Place E. Batailzon, \\ 34060 Montpelzier Cedex, France \\ * Laboratoire de Chimie Physique \\ * Centre d'Etudes d'Electronique des solides
}

\begin{abstract}
We propose a model which explains the evolution of the frequency dependent conductivity while an organized structure appears in an initially amorphous system. We assume that the cristalline organization is connected with the existence of an anisotropy. An anisotropy parameter is calculated. An "a prioni". calculation of the conductivity is developped, which correctly accounts for the experimental results obtained on "Iow temperature" carbons.
\end{abstract}

Introduction.- The theoretical study of amorphous solids has widely used the concept of localized states bound to the existence of a random potential in these materials. The numbering of sites (donors and acceptors) among which the electrons move in an electric field can be obtained by observing the behaviour of conductivity as a function of frequency $(1,2)$. The interpretation of results thus obtained required an implement of a set of models $(3,4)$ based, for most of them, on the assumption that the solid can be represented by a population of localized states (5). Some authors $(6,7)$ have described the conduction mechanism in these materials by modelling the random path of electrons : thus the hint to create simulations (8) using percolations methods (9). All these models contribute to point out a conductivity law as a function of frequency identical to the experimental law $\sigma(\omega) \omega \omega$.

Some works have reported metal-non-metal transitions (2) due to a gradual reorganization of the crystal structure of the compounds. It seems interesting to consider a description of these transitions by trying to establish a correlation between the $s$ exponent, that characterizes the behaviour of conduction as a function of frequency, and the degree of structure organization, measured with the help of an order parameter as described by Landau (10). By doing so, we start f::om à model, describing the properties of amorphous compounds, which contains parameters capable of introducing gradually the concept of order.

I - Proposed model.- Generally it is considered that the properties of amorphous solids are explained with the help of isotropic models. Thus the way to consider the appearance of an order consists in introducing an anisotropy factor into the crystal structure. The dielectric constant becomes a tensor whose three components $\varepsilon_{x}$, $\varepsilon_{y}$, $\varepsilon_{z}$ can be determined. Consequently the potential around an atom must also be anisotropic, which implies that, for a given normal mode the phonons frequency changes according to the considered direction (11), that is $\nu_{x}, \nu_{y}, \nu_{z}$. For the sake of sinplicity, we have supposed that $\varepsilon_{\mathrm{x}}=\varepsilon_{\mathrm{y}}$ (thus $\nu_{\mathrm{x}}=\nu_{\mathrm{y}}$ ). Thus the dielectric constant anisotropy induces a distorsion of equipotential surfaces around a donor site in this model. The surface becomes ellipsoid which large axis, i.e. parallel to $0 z$, has a length $2 a$, and which short axis has a length $2 b$, for a given value $W$, of the energy. $W$, represents the height of the potential barrier that an electron settled in a donor site at the center of the ellipsoĩd must overcome to reach a site at a distance $\mathrm{R}$ on the ellipsoid. It is due to the coulombian interaction forces $(12,13)$. If we cal1 $W_{M}$ the energy required to extract an electron from a donor site (3) we obtain : $W_{M}-W_{X}=e^{2}\left(b \varepsilon_{X}\right)^{-1} ; W_{M}-W_{z}=e^{2}\left(a \varepsilon_{z}\right)^{-1} ;$ and along a direction making an angle $\theta$ with the $O x$ axis, $W_{M}-W_{\theta}=e^{2}\left(R_{\theta} \varepsilon_{\theta}\right)-1, R_{\theta}$ and $\varepsilon_{\theta}$ being the values of the radius vector and of the permittivity along the given direction. Obviously, on an equipotential surface, we have : $W=W_{R}, \theta=W_{x}=W_{y}$ or $\varepsilon_{z} / \varepsilon_{x}=b / a$. When an electric fieid $\&$ of circular frequency $\omega$ is applied, which direction makes an angle $\phi$ with the $0 x$ axis, each pair of sites is located by $\gamma$ (angle with the field direction) and $R$ (distance bet- 
ween both sites). Under these conditions, the pairs of sites thus characterized participate to the conductivity. The real part is (14) :

$$
\frac{i(\omega)}{\varepsilon(\omega)}=\frac{\mathfrak{n}(\gamma, R) \mathrm{e}^{2} \mathrm{R}^{2} \cos ^{2} \gamma}{4 \mathrm{kTch}(W / 2 \mathrm{kT})}-\frac{\omega^{2} \tau}{1+\omega^{2} \tau^{2}}
$$

$i(\omega)$ represents the current density, $n(Y, R)$ is the number of identical pairs of sites and $\tau$ is the relaxation time corresponding to electron hop.

Let $d p(R, \gamma)$ be the number of pairs in the elementary volume dV ; genera1lizing relation (1) leads to the expression of the real elementary conductivity, (assuming that $\left.\operatorname{ch}^{2}(\mathrm{~W} / 2 \mathrm{kT}) \approx 1(5,14)\right)$ :

$$
d \sigma^{\prime}(\omega)=d p(\gamma, R) e^{2} R^{2} \cos ^{2} \gamma(4 k T)^{-1} \omega^{2} \tau\left(1+\omega^{2} \tau^{2}\right)^{-1}
$$

Let $\mathrm{N}_{\mathrm{A}}$ and $\mathrm{N}_{\mathrm{D}}$ be the number of donors and acceptors sites per unit volume, we find the distribution $\mathrm{dp}(Y, R)$ of the number of pairs in the elementary volume $d V: d p(Y, R)=N_{A} N_{D} d V$. The quantity $d V$ is the volume determined by the variations $\mathrm{d} \theta$ and $\mathrm{d} \psi(\psi$ is the angle of rotation of the ellipsoid around the $Z$ axis), and between two equipotential ellipsoidal surfaces, both centered on the donor site and characterized by the semi-axes $a$ and $a+d a$. Using the parametric form of the ellipsoid in cylindric coordinates we obtain: $d V=a^{2}\left(\varepsilon_{z} / \varepsilon_{x}\right)^{2} d a \sin \beta d B d \psi$, where $\beta$ is related to $\theta$ by the equation a $\cos \beta=R \cos \theta$. The appearance of an ordered structure in an initially amorphous solid generally begins by short distance order around localized sites (15). Gradually these sites can be changed into conducting microdomains in which the electronic energy levels can be considered as delocalized (16). However, at the beginning of this evolution, $i . e$. before the appearance of a long distance order, these organized microdomains are still randomly oriented in the material (2). This phenomenon is taken into account by considering the mean value of $\mathrm{do}^{\prime}(\omega)$, in the whole space. Thus, we obtain the mean contribution to the conductivity of dipoles which acceptor site is between two homothetic ellipsoidal surfaces centered on the donor site :

$$
\mathrm{d} \sigma^{\prime}(\omega)={ }_{\mathrm{A}} \mathrm{N}_{\mathrm{D}} \mathrm{e}^{2}(36 \mathrm{kT})^{-1}\left(\varepsilon_{\mathrm{z}} / \varepsilon_{\mathrm{x}}\right)^{2}\left(1+2\left(\varepsilon_{\mathrm{z}} f \varepsilon_{\mathrm{x}}\right)^{2}\right) \omega^{2} \tau\left(1+\omega^{2} \tau^{2}\right)^{-1} \mathrm{a}^{4} \mathrm{da}
$$

The calculated conductivity $\mathrm{d}^{\prime}(\omega)$, is the mean contribution to the conductivity of all dipoles characterized by a hopping time between $\tau$ and $\tau+d \tau$. The relaxation time in the $z$ direction is $: \tau=v_{z}^{-1} \exp (2 \alpha a)$, where $\alpha^{-1}$ represents the Bohr radius of the localized sites. The value da $=(2 \alpha \tau)^{-1} \mathrm{~d} \tau$, introduced in equation (3) gives the conductivity $\sigma^{\prime}(\omega)$ :

$$
\sigma^{\prime}(\omega)=\mathrm{N}_{\mathrm{A}} \mathrm{N}_{\mathrm{D}}(72 \alpha \mathrm{kT})^{-1} \mathrm{e}^{2}\left(\varepsilon_{z} / \varepsilon_{\mathrm{x}}\right)^{2}\left(1+2\left(\varepsilon_{z} / \varepsilon_{\mathrm{x}}\right)^{2}\right) \omega \int_{0}^{\infty} \mathrm{a}^{4} \omega \tau\left(1+\omega^{2} \tau^{2}\right)^{-1} \mathrm{~d} \tau / \tau
$$

The integral can be calculated $(3,4,5,11)$ :

$$
\sigma^{\prime}(\omega)=\pi \mathrm{N}_{\mathrm{A}} \mathrm{N}_{D}(144 \mathrm{kT})^{-1} e^{2}\left(\varepsilon_{z} / \varepsilon_{\mathrm{X}}\right)^{2}\left(1+2\left(\varepsilon_{z} / \varepsilon_{\mathrm{X}}\right)^{2}\right) \omega \mathrm{a}_{\omega}^{4}
$$

where $a_{\omega}$ is the longer semi-axis of the ellipsoid such that the relaxation time corresponding to the hop from the donor site (at the center of the ellipsoĩd) to the acceptor site located on the equipotential surface is $\tau_{\omega}$, with along the $z$ direction $\omega \tau_{\omega}=1$. The relaxation time previously defined leads to $: \omega=\tau^{-1}=v_{\bar{z}} \exp \left(-2 \alpha a_{\omega}\right)$. The value of $a_{\omega}$ can thus be evaluated : $a_{\omega}=(2 \alpha)^{-1} \ln \left(\nu_{z} / \omega\right)$. The investigated expression is written :

$$
\sigma^{\prime}(\omega)=\pi N_{A} N_{D}(72 k T)^{-1}(2 \alpha)^{-5} e^{2}\left(\varepsilon_{z} / \varepsilon_{x}\right)^{2}\left(1+2\left(\varepsilon_{z} / \varepsilon_{x}\right)^{2}\right) \omega\left(\ln \left(\nu_{z} / \omega\right)\right)^{4}
$$

The random orientation of microdomains gives rise to any orientation of the axis of the various ellipsoids that characterize the short distance organization of each donor site. Consequently, the only parameter bound to phonons, and experimentally acceptable, is the mean frequency $\nu_{\mathrm{ph}}(17): \nu_{\mathrm{ph}}^{2}=\frac{1}{3}\left(\nu_{\mathrm{x}}^{2}+v_{\mathrm{y}}^{2}+v_{z}^{2}\right)=\frac{1}{3} \cdot\left(\nu_{\mathrm{z}}^{2}+2 v_{\mathrm{x}}^{2}\right)$, avec $v_{x}=v_{y}$. Let us call $n=v_{x}^{2} / v^{2}$, which allows to ${ }^{3}$ establish a measurement of the anisotropy. It is equal to 1 for an isotropic structure which, in our model, represents the amorphous state, and it takes very high values for a very anisotropic structure, i.e. an ordered structure $(1 \leqslant \eta \leqslant \infty)$. The expression of $\nu_{\mathrm{ph}}$ gives :
$v_{z}=v_{\mathrm{ph}}\left(\frac{1}{3}(1+2 \eta)\right)^{-1 / 2}$. Calling $\mathrm{d}=\left(\frac{1}{3}(1+2 \eta)\right)^{-1 / 2}$ one obtains $\nu_{z}=\mathrm{d} \nu_{\mathrm{ph}}$. The quantity $\mathrm{d}$, is equal to 1 when $n=1$, that is when the structure is totally disordered and tends to $O$ when the system is ordered. Thus this parameter can be related to an anisotropy parameter (10). In fact, the latter must be equal to zero for the most symetric phase and different from zero in the least symetric phase. With $p=1-\mathrm{d}^{2}$, we 
define an anisotropy parameter that meets this criterion capable to describe a transition due to the appearance of an ordered structure in an amorphous material :

$$
\mathrm{p}=\left(\nu_{\mathrm{x}}^{2}-\nu_{\mathrm{z}}^{2}\right)\left\{\nu_{\mathrm{x}}^{2}\left(1+\left(\nu_{\mathrm{z}}^{2} / 2 \nu_{\mathrm{x}}^{2}\right)\right)\right\}^{-1} \text { with } \nu_{\mathrm{x}} \geqslant \nu_{z}
$$

Taking into account $\nu_{z}=d \nu_{p h}$, the equation (2) leads to an equation containing $d$ :

$$
\sigma^{\prime}(\omega)=\pi \mathrm{N}_{\mathrm{A}} \mathrm{N}_{\mathrm{D}}(72 \mathrm{kT})^{-1}(2 \alpha)^{-5} \mathrm{e}^{2}\left(\varepsilon_{\mathrm{z}} / \varepsilon_{\mathrm{x}}\right)^{2}\left(1+2\left(\varepsilon_{\mathrm{z}} / \varepsilon_{\mathrm{x}}\right)^{2}\right) \omega\left(\ln \left(\mathrm{d} \nu_{\mathrm{ph}} / \omega\right)\right)^{4}
$$

Case 1. Isotropic : $\eta=1$ or $\mathrm{d}=1$.

The solid corresponds to a totally disordered material, i.e. amorphous material. Using the approximation suggested by MOTT $(4,5), \mathrm{N}_{A}=\mathrm{N}_{\mathrm{D}}=\operatorname{kTN}\left(\mathrm{E}_{\mathrm{F}}\right)$, we find an expression identical (if we except the constant value) to the equation generally proposed for amorphous compounds :

$$
\begin{aligned}
& \text { compounds : } \\
& \sigma^{\prime}(\omega)=(\pi / 3) \operatorname{kT}\left(\mathrm{N}\left(\mathrm{E}_{\mathrm{F}}\right)\right)^{2} \alpha^{-5} \mathrm{e}^{2} \omega\left(2 \mathrm{n}\left(v_{\mathrm{ph}} / \omega\right)\right)^{4}
\end{aligned}
$$

Under these conditions it has been shown (14) that the real part of the conductivity $\sigma^{\prime}(\omega)$ varies as $\omega^{3}$, that is :

$$
\omega\left(\ln \left(d v_{p h} / \omega\right)\right)^{4}=C \omega^{s}
$$

where $C$ is a constant. Studying this function leads to a value of $s$ of the form (4):

$$
\mathrm{s}=1-4 / \ell \mathrm{n}\left(\frac{\mathrm{d} \nu_{\mathrm{p}} \mathrm{h}}{\omega}\right)
$$
One can see that in the case where $\mathrm{d} \nu_{\mathrm{ph}} / \omega$ is $\stackrel{\omega}{s}$ large (with $\mathrm{d}=1$ ), which is always ex-
perimentally verified, $s \rightarrow 1$.

Case 2. n large, d sma11.

For a given phonon mean frequency $v_{p h}$, if d decreases, $d \nu_{p h} / \omega$ is such that according to equation (5), $s \rightarrow 0$. The conductivity becomes independent of the frequency. Between these two limiting cases, $s$ is a decreasing uniform monotonous function of $d$ in the interval 10,1$]$.

II - Experimental verification. We have used the frequency dependent conductivity of "low temperature carbons" around a non-metal-metal transition (2). In fig. 1 we have drawn the conductivity as a function of frequency (logarithmic scale, $\mathrm{T}=186 \mathrm{~K}$ ) for 3 carbons of heat treatment temperature (HTT) 600,650 and $730^{\circ} \mathrm{C}$. The values of $\mathrm{s}$ are $0.77,0.61$ and 0 , respectively (2). We observe that $s \rightarrow 0$ when HTT increases. Theses carbons are gradually organized when HTT increases, i.e. the dimension of perfectly organized crystallites increases regularly (18). This result can be considered as a rather satisfactory experimental picture of the behaviour foreseen by the model proposed.

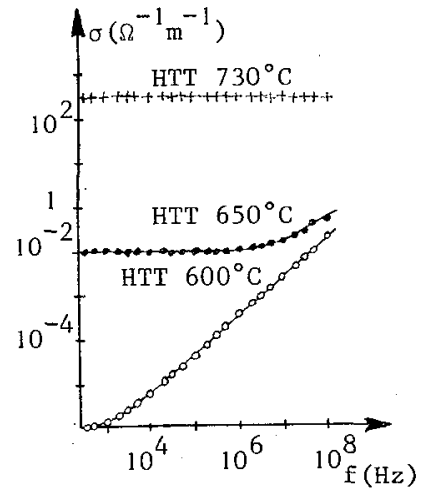

Fig. 1 - Frequency dependence of the conductivity of 3 carbons $(\mathrm{T}=186 \mathrm{~K})$ with increasing HTT.

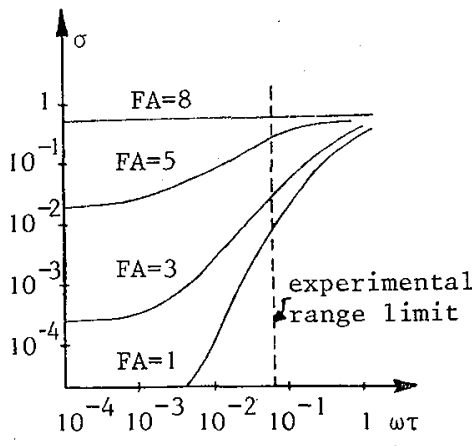

Fig.2-Simulated frequency dependent conductivity as a function of the anisotropy factor FA.

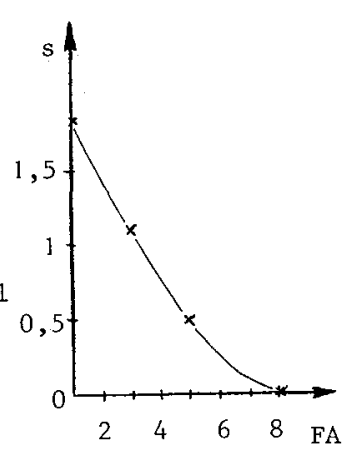

Fig.3-Effect of the anisotropy factor FA, on the exponent $s$.

III - Calculation of the conductivity in an anisotropic model. To simulate numerical1y the proposed model we used a method of percolation. The lattice used here is derwed from previous works $(19,20)$ and is a square two dimensional $30 \times 30$ mode lattice. Each node is bound to its neighbours by orthogonal bounds. The bound is considered as a good or a poor conductor according to the value of $r$ : if $r$ is greater than a 
given threshold $r_{0}$ formally defined, the bound is considered as a good conductor and is then represented by a resistor $R_{0}$. If not, it is considered as a poor conductor and represented by a resistor $R_{1}$ and a capacitor $C_{1}$ in parallel. In this work, the ratio $R_{1} / R_{0}$ is taken equal to $10^{4}$. We have simulated the anisotropy of the material by introducing an anisotropic factor FA, as follows : every bound of the lattice is scanned one after the other. Each time we meet a conducting bound B, it is extended by FA-1 conducting bounds along the direction perpendicular to the electrodes, independantly of the previous values of these bounds. Thus we introduce into the lattice sequences of FAconducting bounds along the direction perpendicular to the electrodes. Obviously FA depends on the extent of organization of the lattice, so that FA $=1$ represents an isotropic structure. We obtain a new complex correlated lattice in which the potential is calculated at each mode by the Gauss-Seidel iteration procedure improved by a relaxation process described el sewhere (21). In order to reduce the computation time, the rate of conducting bounds is set to $15.3 \%$. Then the conductivity of the simulated material is known for a given voltage and frequency. Drawing $\log 0$ as a function of $\log \omega$ for various values of the anisotropic factor, we obtain the results shown in fig.2. We observe a noteworthy likeness between the family of experimental curves (fig.i) and the curves obtained by simulation (fig.2). Moreover we find the experimental evolution of $s$ (fig.3). The high values found for $s$, in an isotropic solid can be explained by the simplicity of the two-dimensional model designed to interpret the behaviour of a three-dimensional solid.

III - Conclusion. Considering the equation (5), we find :

$$
s=1-4 / \ln \left(\frac{v p h(1-p) i / 2}{\omega}\right)
$$

where $p$ is the anisotropy parameter formally defined (equation 4). The proposed model leads to a relation that binds explicitely the $s$ exponant to a structural parameter $p$, on a large range of frequencies. p characterizes some insulator-semiconductor transitions associated to the gradual appearance of an order in initially amorphous solids. The $s$ parameter, that can be directly obtained experimentally is aconvenient way to evaluate the order in some cases.

\section{References.}

(1) A.E. OWEN and J.M. ROBERTSON - J. Non Crystaliine Solids 2 (1970) 40 .

(2) J.C. GIUNTINI, D. JULLIEN, J.V. ZANCHETTA, F. CARMONA and P. DELHAES - J. NonCrystalline Solids 30 (i978) 87.

(3) S.R. ELLIOTT - Phil. Mag. 36 (1977) 1291.

(4) N.F. MOTT and E.A. DAVIS - Electronic Processes in Non Crystallines Materials (C1arendon Oxford 1971).

(5) M. POLLAK - Phil. Mag. 23 (1971) 519.

(6) A.K. JONSCHER - J. Non-Crystalline Solids 8 (1972) 293.

(7) H. SCHER and E.W. MONTROLL - Phys. Rev. B1 $\overline{2}$ (1975) 2455.

(8) M. POLLAK and I. RIESS - Phys. Stat. Sol $\overline{\text { B } 69}$ (1975) K15.

(9) G.E. PIKE and C.H. SEAGER - Phys. Rev. B10 (1974) 1421.

(10) L. LANDAU and E. LIFCHIT2 - Physique Statistique (Editions MIR Moscou 1967).

(11) C. KITTEL - Théorie Quantique du Solide - Dunod - Paris (1967).

(12) S.R. ELLIOTT - Solid St. Commun. 27 (1978) 749.

(13) G.E. PIKE - Phys. Rev. B6 (1972) 1572 .

(14) M. POLLAK and T.H. GEBAL̄LE - Phys. Rev. 122 (1961) 1742.

(15) J.M. MACKOWSKI, J.J. SAMUELLI, P. KUMURD $\overline{J A N}$ and BOURDARIOS - J. Non-Crystalline Solids 8 (1972) 985 .

(16) E.N. ECŌNOMOU, M.H. COHEN, K.F. FREED and E.S. KIRPPATRICK - Amorphous and Liquïd Semiconductors p. 45. Plenum Press (1974) Londres.

(17) H. BROOKS - Advances in electronics and electrons Physics 7 (1955) 85.

(18) F. CARMONA and P. DELHAES - J. App1. Phys. 49 (1978) 618.

(19) J.L. JACQUEMIN, A. ARDALAN and G. BORDURE - J. Non-Crystalline Solids 28 (1978)249.

(20) M.H. COHEN and J. JORTNER - Phys. Rev. Lett. 30 (1973) 696.

(21) H.J. JURETSCHKE, R. LANDAUER, J.A. SNANSON - J. App1. Phys. 27 (1956) 838. 